\title{
Fixed Point Theorems for a Class of $\alpha$-Admissible Contractions and Applications to Boundary Value Problem
}

\author{
Hamed H. Alsulami, ${ }^{1}$ Selma Gülyaz, ${ }^{2}$ Erdal Karapınar, ${ }^{1,3}$ and İncı M. Erhan ${ }^{3}$ \\ ${ }^{1}$ Nonlinear Analysis and Applied Mathematics (NAAM) Research Group, King Abdulaziz University, Jeddah, Saudi Arabia \\ ${ }^{2}$ Department of Mathematics, Cumhuriyet University, Sivas, Turkey \\ ${ }^{3}$ Department of Mathematics, Atilim University, Incek, 06836 Ankara, Turkey
}

Correspondence should be addressed to Erdal Karapınar; erdalkarapinar@yahoo.com

Received 15 May 2014; Accepted 12 June 2014; Published 3 July 2014

Academic Editor: Jen-Chih Yao

Copyright (C) 2014 Hamed H. Alsulami et al. This is an open access article distributed under the Creative Commons Attribution License, which permits unrestricted use, distribution, and reproduction in any medium, provided the original work is properly cited.

A class of $\alpha$-admissible contractions defined via altering distance functions is introduced. The existence and uniqueness conditions for fixed points of such maps on complete metric spaces are investigated and related fixed point theorems are presented. The results are reconsidered in the context of partially ordered metric spaces and applied to boundary value problems for differential equations with periodic boundary conditions.

\section{Introduction and Preliminaries}

Recent developments in fixed point theory have shown the significance of theoretical studies which are directly applicable in other areas. In particular, the problems related with existence and uniqueness of solutions of integral and differential equations are of particular importance. Differential and integral equations govern the behaviour of various real-life problems for which the question of existence and uniqueness of solutions is crucial. This fact motivates the intensive research activities in the area and the rapidly increasing number of publications [1-7].

The main goal of studies in fixed point theory is to improve the contractive conditions imposed on the mappings under consideration. Altering distance functions defined by Khan et al. [8] have been widely used for this reason both alone or combined with other auxiliary functions.

Definition 1. An altering distance function is a function $\psi$ : $[0,+\infty) \rightarrow[0,+\infty)$ which satisfies the following:

(1) $\psi$ is continuous and nondecreasing;

(2) $\psi(t)=0 \Leftrightarrow t=0$.

Admissible mappings have been defined recently by Samet et al. [6] and employed quite often in order to generalize the results on various contractions. We state next the definitions of $\alpha$-admissible mapping and triangular $\alpha$ admissible mappings.

Definition 2. A mapping $T: X \rightarrow X$ is called $\alpha$-admissible if for all $x, y \in X$ one has

$$
\alpha(x, y) \geq 1 \Longrightarrow \alpha(T x, T y) \geq 1
$$

where $\alpha: X \times X \rightarrow[0, \infty)$ is a given function.

Definition 3. A mapping $T: X \rightarrow X$ is called triangular $\alpha$ admissible if it is $\alpha$-admissible and satisfies

$$
\begin{aligned}
& \alpha(x, y) \geq 1 \\
& \alpha(y, z) \geq 1
\end{aligned} \Longrightarrow \alpha(x, z) \geq 1
$$

where $x, y, z \in X$ and $\alpha: X \times X \rightarrow[0, \infty)$ is a given function.

Inspired by this definition, we define the following weaker condition which proves to be sufficient in the forthcoming discussions.

Definition 4. A mapping T:X $\rightarrow X$ is said to be weak triangular $\alpha$-admissible if it is $\alpha$-admissible and satisfies

$$
\alpha(x, T x) \geq 1 \Longrightarrow \alpha\left(x, T^{2} x\right) \geq 1,
$$

where $x \in X$ and $\alpha: X \times X \rightarrow[0, \infty)$ is a given function. 
Weak triangular $\alpha$-admissible mappings satisfy a property stated in the following lemma and the proof of which easily follows from the definition and can be found in [9].

Lemma 5 (see [9]). Let $T: X \rightarrow X$ be a weak triangular $\alpha$-admissible mapping. Assume that there exists $x_{0} \in X$ such that $\alpha\left(x_{0}, T x_{0}\right) \geq 1$. If $x_{n}=T^{n} x_{0}$, then $\alpha\left(x_{m}, x_{n}\right) \geq 1$ for all $m, n \in \mathbb{N}$ with $m<n$.

\section{Existence and Uniqueness Theorems on Complete Metric Spaces}

In this section we present our main results which include theorems on existence and uniqueness of fixed points for a class of weak triangular $\alpha$-admissible mappings.

First we define the following two classes of contractions which we are going to investigate in this section and throughout the paper.

Definition 6. Let $(X, d)$ be a metric space, $\psi$ an altering distance function, and $\phi:[0,+\infty) \rightarrow[0,+\infty)$ a continuous function satisfying $\psi(t)>\phi(t)$ for all $t>0$.

(I) A mapping $T: X \rightarrow X$ belongs to class (I) if it satisfies

$$
\alpha(x, y) \psi(d(T x, T y)) \leq \phi(M(x, y)), \quad \forall x, y \in X,
$$

where

$$
\begin{array}{r}
M(x, y)=\max \{d(x, y), d(x, T x), d(y, T y), \\
\left.\frac{1}{2}[d(x, T y)+d(y, T x)]\right\} .
\end{array}
$$

(II) A mapping $T: X \rightarrow X$ belongs to class (II) if it satisfies

$$
\alpha(x, y) \psi(d(T x, T y)) \leq \phi(N(x, y)), \quad \forall x, y \in X,
$$

where

$$
\begin{aligned}
N(x, y)=\max \left\{d(x, y), \frac{1}{2}[d(x, T x)+d(y, T y)],\right. & \left.\frac{1}{2}[d(x, T y)+d(y, T x)]\right\} .
\end{aligned}
$$

Remark 7. Note that $N(x, y) \leq M(x, y)$ for all $x, y \in X$.

Our first theorem gives conditions for the existence of a fixed point for maps in class (I).

Theorem 8. Let $(X, d)$ be a complete metric space. Let $T$ : $X \rightarrow X$ be a continuous, weak triangular $\alpha$-admissible mapping such that

$$
\alpha(x, y) \psi(d(T x, T y)) \leq \phi(M(x, y)), \quad \forall x, y \in X,
$$

where $\psi$ is an altering distance function, $\phi:[0,+\infty) \rightarrow$ $[0,+\infty)$ is a continuous function satisfying $\psi(t)>\phi(t)$ for all $t>0$, and $M(x, y)=\max \{d(x, y), d(x, T x), d(y, T y)$, $(1 / 2)[d(x, T y)+d(y, T x)]\}$. If there exists $x_{0} \in X$ such that $\alpha\left(x_{0}, T x_{0}\right) \geq 1$, then $T$ has a fixed point.

Proof. Let $x_{0} \in X$ satisfy $\alpha\left(x_{0}, T x_{0}\right) \geq 1$ and define the sequence $\left\{x_{n}\right\}$ as $x_{n}=T x_{n-1}$ for $n \in \mathbb{N}$.

If $x_{n_{0}}=x_{n_{0}+1}$ for some $n_{0} \in \mathbb{N}$, then, obviously, $x_{n_{0}}=T x_{n_{0}}$ is a fixed point of $T$. Suppose that $d\left(x_{n}, x_{n+1}\right)>0$ for all $n \in \mathbb{N}$.

Note that, due to the fact that $T$ is $\alpha$-admissible and $\alpha\left(x_{0}, T x_{0}\right)=\alpha\left(x_{0}, x_{1}\right) \geq 1$, we deduce

$$
\begin{aligned}
& \alpha\left(T x_{0}, T x_{1}\right) \\
& \quad=\alpha\left(x_{1}, x_{2}\right) \geq 1 \Longrightarrow \alpha\left(T x_{1}, T x_{2}\right) \\
& \quad=\alpha\left(x_{2}, x_{3}\right) \geq 1 \Longrightarrow \cdots \Longrightarrow \alpha\left(x_{n}, x_{n+1}\right) \geq 1 .
\end{aligned}
$$

Substituting $x=x_{n}$ and $y=x_{n-1}$ in (8) and using (9) we get

$$
\begin{aligned}
\psi\left(d\left(x_{n+1}, x_{n}\right)\right) & \leq \alpha\left(x_{n}, x_{n-1}\right) \psi\left(d\left(x_{n+1}, x_{n}\right)\right) \\
& =\alpha\left(x_{n}, x_{n-1}\right) \psi\left(d\left(T x_{n}, T x_{n-1}\right)\right) \\
& \leq \phi\left(M\left(x_{n}, x_{n-1}\right)\right),
\end{aligned}
$$

where

$$
\begin{aligned}
& M\left(x_{n}, x_{n-1}\right) \\
& =\max \left\{d\left(x_{n}, x_{n-1}\right), d\left(x_{n}, T x_{n}\right), d\left(x_{n-1}, T x_{n-1}\right),\right. \\
& \left.\quad \frac{1}{2}\left[d\left(x_{n}, T x_{n-1}\right)+d\left(x_{n-1}, T x_{n}\right)\right]\right\} \\
& =\max \left\{d\left(x_{n}, x_{n-1}\right), d\left(x_{n}, x_{n+1}\right), d\left(x_{n-1}, x_{n}\right),\right. \\
& \left.\quad \frac{1}{2}\left[d\left(x_{n}, x_{n}\right)+d\left(x_{n-1}, x_{n+1}\right)\right]\right\} \\
& =\max \left\{d\left(x_{n}, x_{n-1}\right), d\left(x_{n}, x_{n+1}\right), \frac{d\left(x_{n-1}, x_{n+1}\right)}{2}\right\} .
\end{aligned}
$$

Note that $d\left(x_{n-1}, x_{n+1}\right) / 2 \leq(1 / 2)\left[d\left(x_{n-1}, x_{n}\right)+d\left(x_{n}, x_{n+1}\right)\right]$ is smaller than both $d\left(x_{n-1}, x_{n}\right)$ and $d\left(x_{n}, x_{n+1}\right)$. Then, $M\left(x_{n}, x_{n-1}\right)$ can be either $d\left(x_{n-1}, x_{n}\right)$ or $d\left(x_{n}, x_{n+1}\right)$. If $M\left(x_{n}, x_{n-1}\right)=d\left(x_{n}, x_{n+1}\right)$ for some $n$, then the expression (10) implies that

$$
0<\psi\left(d\left(x_{n+1}, x_{n}\right)\right) \leq \phi\left(d\left(x_{n}, x_{n+1}\right)\right),
$$

which contradicts the condition $\psi(t)>\phi(t)$ for $t>0$. Hence $M\left(x_{n}, x_{n-1}\right)=d\left(x_{n}, x_{n-1}\right)$ for all $n \geq 1$ and we have

$$
0<\psi\left(d\left(x_{n+1}, x_{n}\right)\right) \leq \phi\left(d\left(x_{n}, x_{n-1}\right)\right)<\psi\left(d\left(x_{n}, x_{n-1}\right)\right),
$$

which results in

$$
d\left(x_{n+1}, x_{n}\right)<d\left(x_{n}, x_{n-1}\right),
$$


since $\psi$ is nondecreasing. Thus, we conclude that the nonnegative sequence $d\left(x_{n+1}, x_{n}\right)$ is decreasing. Therefore, there exists $r \geq 0$ such that $\lim _{n \rightarrow \infty} d\left(x_{n+1}, x_{n}\right)=r$. Let $n \rightarrow \infty$ in (10); we get

$$
\psi(r) \leq \phi(r) .
$$

By the hypothesis of the theorem, since $\psi(t)>\phi(t)$, for all $t>0$, this inequality is possible only if $r=0$, and hence

$$
\lim _{n \rightarrow \infty} d\left(x_{n+1}, x_{n}\right)=r=0
$$

Next, we will prove that $\left\{x_{n}\right\}$ is a Cauchy sequence. Suppose, on the contrary, that $\left\{x_{n}\right\}$ is not Cauchy. Then, for some $\varepsilon>0$, there exist subsequences $\left\{x_{m_{k}}\right\}$ and $\left\{x_{n_{k}}\right\}$ of $\left\{x_{n}\right\}$ such that

$$
n_{k}>m_{k}>k, \quad d\left(x_{n_{k}}, x_{m_{k}}\right) \geq \varepsilon
$$

for all $k \geq 1$, where, corresponding to each $m_{k}$, we can choose $n_{k}$ as the smallest integer with $n_{k}>m_{k}$ for which (17) holds. Then

$$
d\left(x_{n_{k}}, x_{m_{k-1}}\right)<\varepsilon
$$

Employing triangle inequality and making use of (17) and (18), we obtain

$$
\begin{aligned}
\varepsilon & \leq d\left(x_{n_{k}}, x_{m_{k}}\right) \\
& \leq d\left(x_{n_{k}}, x_{n_{k-1}}\right)+d\left(x_{n_{k-1}}, x_{m_{k}}\right) \\
& <d\left(x_{n_{k}}, x_{n_{k-1}}\right)+\varepsilon .
\end{aligned}
$$

Passing to limit as $k \rightarrow \infty$ and using (16), we get

$$
\lim _{k \rightarrow \infty} d\left(x_{n_{k}}, x_{m_{k}}\right)=\varepsilon
$$

From the triangular inequality, we also have

$$
\begin{aligned}
& d\left(x_{n_{k}}, x_{m_{k}}\right) \\
& \quad \leq d\left(x_{n_{k}}, x_{n_{k-1}}\right)+d\left(x_{n_{k-1}}, x_{m_{k-1}}\right)+d\left(x_{m_{k-1}}, x_{m_{k}}\right), \\
& d\left(x_{n_{k-1}}, x_{m_{k-1}}\right) \\
& \quad \leq d\left(x_{n_{k-1}}, x_{n_{k}}\right)+d\left(x_{n_{k}}, x_{m_{k}}\right)+d\left(x_{m_{k}}, x_{m_{k-1}}\right) .
\end{aligned}
$$

Letting $k \rightarrow \infty$ in the two inequalities above and using (16) and (20), we get

$$
\lim _{k \rightarrow \infty} d\left(x_{n_{k-1}}, x_{m_{k-1}}\right)=\varepsilon
$$

In a similar way, by using the triangular inequality, we obtain that

$$
\begin{aligned}
& d\left(x_{n_{k}}, x_{m_{k}}\right) \leq d\left(x_{n_{k}}, x_{n_{k-1}}\right)+d\left(x_{n_{k-1}}, x_{m_{k}}\right), \\
& d\left(x_{n_{k-1}}, x_{m_{k}}\right) \leq d\left(x_{n_{k-1}}, x_{n_{k}}\right)+d\left(x_{n_{k}}, x_{m_{k}}\right) .
\end{aligned}
$$

Taking limit as $k \rightarrow \infty$ in the above two inequalities and regarding (16) and (20), we get

$$
\lim _{k \rightarrow \infty} d\left(x_{n_{k-1}}, x_{m_{k}}\right)=\varepsilon
$$

Furthermore, the relations

$$
\begin{aligned}
& d\left(x_{n_{k}}, x_{m_{k}}\right) \leq d\left(x_{n_{k}}, x_{m_{k-1}}\right)+d\left(x_{m_{k-1}}, x_{m_{k}}\right), \\
& d\left(x_{n_{k}}, x_{m_{k-1}}\right) \leq d\left(x_{n_{k}}, x_{n_{k-1}}\right)+d\left(x_{n_{k-1}}, x_{m_{k-1}}\right)
\end{aligned}
$$

give

$$
\lim _{k \rightarrow \infty} d\left(x_{n_{k}}, x_{m_{k-1}}\right)=\varepsilon
$$

by letting $k \rightarrow \infty$ and taking into account (16) and (20). By the definition of $M(x, y)$ and using limits found above, we get

$$
\lim _{k \rightarrow \infty} M\left(x_{n_{k-1}}, x_{m_{k-1}}\right)=\varepsilon
$$

Indeed, since

$$
\begin{aligned}
& M\left(x_{n_{k-1}}, x_{m_{k-1}}\right) \\
& =\max \left\{d\left(x_{n_{k-1}}, x_{m_{k-1}}\right), d\left(x_{n_{k-1}}, T x_{n_{k-1}}\right),\right. \\
& d\left(x_{m_{k-1}}, T x_{m_{k-1}}\right), \\
& \left.\quad \frac{1}{2}\left[d\left(x_{n_{k-1}}, T x_{m_{k-1}}\right)+d\left(x_{m_{k-1}}, T x_{n_{k-1}}\right)\right]\right\} \\
& =\max \left\{d\left(x_{n_{k-1}}, x_{m_{k-1}}\right), d\left(x_{n_{k-1}}, x_{n_{k}}\right), d\left(x_{m_{k-1}}, x_{m_{k}}\right),\right. \\
& \left.\frac{1}{2}\left[d\left(x_{n_{k-1}}, x_{m_{k}}\right)+d\left(x_{m_{k-1}}, x_{n_{k}}\right)\right]\right\},
\end{aligned}
$$

passing to the limit as $k \rightarrow \infty$ in (28) and using (16), (20), (22), (24), and (26), we obtain

$$
\lim _{n \rightarrow \infty} M\left(x_{n_{k-1}}, x_{m_{k-1}}\right)=\max \left\{\varepsilon, 0,0, \frac{1}{2}[\varepsilon+\varepsilon]\right\}=\varepsilon .
$$

Notice that since $T$ is weak triangular $\alpha$-admissible, we deduce from Lemma 5 that $\alpha\left(x_{n_{k-1}}, x_{m_{k-1}}\right) \geq 1$. Therefore, we can apply condition (8) to $x_{n_{k-1}}$ and $x_{m_{k-1}}$ to obtain

$$
\begin{aligned}
0 & <\psi\left(d\left(x_{n_{k}}, x_{m_{k}}\right)\right) \\
& \leq \alpha\left(x_{n_{k}-1}, x_{m_{k}-1}\right) \psi\left(d\left(x_{n_{k}}, x_{m_{k}}\right)\right) \\
& \leq \phi\left(M\left(x_{n_{k-1}}, x_{m_{k-1}}\right)\right) .
\end{aligned}
$$

Letting $k \rightarrow \infty$ and taking into account (20) and (27), we have

$$
0<\psi(\varepsilon) \leq \phi(\varepsilon) .
$$

However, since $\psi(t)>\phi(t)$, for $t>0$, we deduce that $\varepsilon=0$, which contradicts the assumption that $\left\{x_{n}\right\}$ is not a Cauchy 
sequence. Thus, $\left\{x_{n}\right\}$ must be Cauchy. Due to the fact that $(X, d)$ is a complete metric space, there exists $u \in X$ such that $\lim _{n \rightarrow \infty} x_{n}=u$. Finally, the continuity of $T$ gives

$$
u=\lim _{n \rightarrow \infty} x_{n}=\lim _{n \rightarrow \infty} T x_{n-1}=T u .
$$

That is, $u$ is a fixed point of $T$, which completes the proof.

One of the advantages of $\alpha$-admissible mappings is that the continuity of the map is no longer required for the existence of a fixed point provided that the space under consideration has the following property.

(A) If $\left\{x_{n}\right\}$ is a sequence in $X$ such that

$$
x_{n} \longrightarrow x, \quad \alpha\left(x_{n}, x_{n+1)}\right) \geq 1 \quad \forall n \in \mathbb{N},
$$

then there exists a subsequence $\left\{x_{n_{k}}\right\}$ of $\left\{x_{n}\right\}$ for which

$$
\alpha\left(x_{n_{k}}, x\right) \geq 1 \quad \forall k \in \mathbb{N} .
$$

Bearing this fact in mind, we rewrite the statement of Theorem 8 in the light of this property.

Theorem 9. Let $(X, d)$ be a complete metric space. Assume that $X$ satisfies condition (A). Let $T: X \rightarrow X$ be a weak triangular $\alpha$-admissible mapping such that

$$
\begin{array}{r}
\alpha(x, y) \psi(d(T x, T y)) \leq \phi(M(x, y)), \\
\forall x, y \in X,
\end{array}
$$

where $\psi$ is an altering distance function, $\phi:[0,+\infty) \rightarrow[0$, $+\infty)$ is a continuous function satisfying $\psi(t)>\phi(t)$ for all $t>0$, and $M(x, y)=\max \{d(x, y), d(x, T x), d(y, T y)$, $(1 / 2)[d(x, T y)+d(y, T x)]\}$. If there exists $x_{0} \in X$ such that $\alpha\left(x_{0}, T x_{0}\right) \geq 1$, then $T$ has a fixed point.

Proof. Following the proof of Theorem 8, it is clear that the sequence $\left\{x_{n}\right\}$ defined by $x_{n}=T x_{n-1}$, for $n \in \mathbb{N}$, converges to a limit $u \in X$. The only thing which remains to show is $T u=u$. Since $\lim _{n \rightarrow \infty} x_{n}=u$, then condition (A) implies $\alpha\left(x_{n_{k}}, u\right) \geq 1$ for all $k \in \mathbb{N}$. Consequently, inequality (35) with $x=x_{n_{k}}$ and $y=u$ becomes

$$
\begin{aligned}
& \psi\left(d\left(x_{n_{k}+1}, T u\right)\right) \\
& \quad \leq \alpha\left(x_{n_{k}}, u\right) \psi\left(d\left(x_{n_{k}+1}, T u\right)\right) \\
& \quad=\alpha\left(x_{n_{k}}, u\right) \psi\left(d\left(T x_{n_{k}}, T u\right)\right) \\
& \quad \leq \phi\left(M\left(x_{n_{k}}, u\right)\right)
\end{aligned}
$$

where

$$
\begin{aligned}
& M\left(x_{n_{k}}, u\right) \\
& =\max \left\{d\left(x_{n_{k}}, u\right), d\left(x_{n_{k}}, x_{n_{k}+1}\right),\right. \\
& \left.\quad d(u, T u), \frac{1}{2}\left[d\left(x_{n_{k}}, T u\right)+d\left(u, x_{n_{k}+1}\right)\right]\right\} .
\end{aligned}
$$

Passing to limit as $k \rightarrow \infty$ and taking into account the continuity of $\psi$ and $\phi$, we get

$$
\psi(d(u, T u)) \leq \phi(d(u, T u)) .
$$

From the condition $\psi(t)>\phi(t)$, for $t>0$, we conclude that $d(u, T u)=0$ and, hence, $T u=u$, which completes the proof.

Similar results can be stated for a map $T: X \rightarrow X$ in the class (II). More precisely, conditions for existence of a fixed point of a map in class (II) are given in the next two theorems.

Theorem 10. Let $(X, d)$ be a complete metric space. Let $T$ : $X \rightarrow X$ be a continuous, weak triangular $\alpha$-admissible mapping such that

$$
\alpha(x, y) \psi(d(T x, T y)) \leq \phi(N(x, y)), \quad \forall x, y \in X,
$$

where $\psi$ is an altering distance function, $\phi:[0,+\infty) \rightarrow$ $[0,+\infty)$ is a continuous function satisfying $\psi(t)>\phi(t)$ for all $t>0$, and $N(x, y)=\max \{d(x, y),(1 / 2)[d(x, T x)+$ $d(y, T y)],(1 / 2)[d(x, T y)+d(y, T x)]\}$. If there exists $x_{0} \in X$ such that $\alpha\left(x_{0}, T x_{0}\right) \geq 1$, then $T$ has a fixed point.

Theorem 11. Let $(X, d)$ be a complete metric space. Assume that $X$ satisfies condition (A). Let $T: X \rightarrow X$ be a weak triangular $\alpha$-admissible mapping such that

$$
\alpha(x, y) \psi(d(T x, T y)) \leq \phi(N(x, y)), \quad \forall x, y \in X,
$$

where $\psi$ is an altering distance function, $\phi:[0,+\infty) \rightarrow$ $[0,+\infty)$ is a continuous function satisfying $\psi(t)>\phi(t)$ for all $t>0$, and $N(x, y)=\max \{d(x, y),(1 / 2)[d(x, T x)+$ $d(y, T y)],(1 / 2)[d(x, T y)+d(y, T x)]\}$. If there exists $x_{0} \in X$ such that $\alpha\left(x_{0}, T x_{0}\right) \geq 1$, then $T$ has a fixed point.

Note that the proofs of Theorems 10 and 11 can be easily done by mimicking the proofs of Theorems 8 and 9, respectively.

We next discuss the conditions for the uniqueness of the fixed point. A sufficient condition for the uniqueness of the fixed point in Theorems 10 and 11 can be stated as follows.

(B)

$$
\begin{aligned}
& \text { For } x, y \in X \text {, there exists } z \in X \\
& \text { such that } \alpha(x, z) \geq 1, \alpha(y, z) \geq 1 \text {. }
\end{aligned}
$$

Note, however, that this condition is not sufficient for the uniqueness of fixed point for maps of class (I).

Theorem 12. If condition $(B)$ is added to the hypotheses of Theorem 10 (resp., Theorem 11), then the fixed point of $T$ is unique.

Proof. Since $T$ satisfies the hypothesis of Theorem 10 (resp., Theorem 11), then fixed point of $T$ exists. Suppose that we have two different fixed points; say $x, y \in X$. From condition (B), there exists $z \in X$, such that

$$
\alpha(x, z) \geq 1, \quad \alpha(y, z) \geq 1 .
$$


Then, since $T$ is $\alpha$-admissible, we have from (42)

$$
\alpha\left(x, T^{n} z\right) \geq 1, \quad \alpha\left(y, T^{n} z\right) \geq 1, \quad \forall n \in \mathbb{N} .
$$

Thus, for the sequence $\left\{z_{n}\right\} \in X$ defined as $z_{n}=T^{n} z$, we have

$$
\begin{aligned}
0 & <\psi\left(d\left(x, z_{n+1}\right)\right) \\
& \leq \alpha\left(x, z_{n}\right) \psi\left(d\left(T x, T z_{n}\right)\right) \\
& \leq \phi\left(N\left(x, z_{n}\right)\right),
\end{aligned}
$$

where

$$
\begin{aligned}
& N\left(x, z_{n}\right) \\
& =\max \left\{d\left(x, z_{n}\right), \frac{1}{2}\left[d(x, T x)+d\left(z_{n}, T z_{n}\right)\right],\right. \\
& \left.\quad \frac{1}{2}\left[d\left(x, T z_{n}\right)+d\left(z_{n}, T x\right)\right]\right\} \\
& =\max \left\{d\left(x, z_{n}\right), \frac{d\left(z_{n}, z_{n+1}\right)}{2},\right. \\
& \left.\frac{1}{2}\left[d\left(x, z_{n+1}\right)+d\left(z_{n}, x\right)\right]\right\} .
\end{aligned}
$$

Observe that $d\left(z_{n}, z_{n+1}\right) / 2 \leq\left(d\left(z_{n}, x\right)+d\left(x, z_{n+1}\right)\right) / 2$. Then we deduce $N(x, y)=\max \left\{d\left(x, z_{n}\right), d\left(x, z_{n+1}\right)\right\}$.

Without loss of generality, we may assume that $d\left(x, z_{n}\right)>$ 0 for all $n \in \mathbb{N}$. If $N(x, y)=d\left(x, z_{n+1}\right)$, then inequality (44) becomes

$$
\begin{aligned}
0 & <\psi\left(d\left(x, z_{n+1}\right)\right) \\
& \leq \alpha\left(x, z_{n}\right) \psi\left(d\left(T x, T z_{n}\right)\right) \\
& \leq \phi\left(d\left(x, z_{n+1}\right)\right) \\
& <\psi\left(d\left(x, z_{n+1}\right)\right) .
\end{aligned}
$$

That is, we have a contradiction. Then we should have $N(x, y)=d\left(x, z_{n}\right)$ for all $n \in \mathbb{N}$, which results in

$$
\begin{aligned}
0 & <\psi\left(d\left(x, z_{n+1}\right)\right) \\
& \leq \alpha\left(x, z_{n}\right) \psi\left(d\left(T x, T z_{n}\right)\right) \\
& \leq \phi\left(d\left(x, z_{n}\right)\right)<\psi\left(d\left(x, z_{n}\right)\right),
\end{aligned}
$$

due to the fact that $\psi(t)>\phi(t)$ for $t>0$. On the other hand, since $\psi$ is nondecreasing, then $d\left(x, z_{n+1}\right) \leq d\left(x, z_{n}\right)$ for all $n \in$ $\mathbb{N}$. Thus, the sequence $\left\{d\left(x, z_{n}\right)\right\}$ is a positive nonincreasing sequence and, hence, converges to a limit; say, $L \geq 0$. Taking limit as $n \rightarrow \infty$ in (47) and regarding continuity of $\psi$ and $\phi$, we deduce

$$
0 \leq \psi(L) \leq \phi(L)
$$

which is possible only if $L=0$. Hence, we conclude that

$$
\lim _{n \rightarrow \infty} d\left(x, z_{n}\right)=0 .
$$

In a similar way, we obtain

$$
\lim _{n \rightarrow \infty} d\left(y, z_{n}\right)=0
$$

From (49) and (50) it follows that $x=y$, which completes the proof of uniqueness.

The theorems stated above have been inspired by the recent results of Yan et al. [7]. They discussed contraction mappings defined on partially ordered complete metric spaces and their applications to boundary value problems. We state next a theorem which can be regarded as a generalization of the main result in [7] in complete metric spaces.

Theorem 13. Let $(X, d)$ be a complete metric space. Let $T$ : $X \rightarrow X$ be a weak triangular $\alpha$-admissible mapping such that

$$
\alpha(x, y) \psi(d(T x, T y)) \leq \phi(d(x, y)), \quad \forall x, y \in X,
$$

where $\psi$ is an altering distance function and $\phi:[0,+\infty) \rightarrow$ $[0,+\infty)$ is a continuous function satisfying $\psi(t)>\phi(t)$ for all $t>0$. Assume either that $T$ is continuous or that $X$ satisfies condition (A). If there exists $x_{0} \in X$ such that $\alpha\left(x_{0}, T x_{0}\right) \geq 1$, then $T$ has a fixed point. If, in addition, $X$ satisfies condition (B), then the fixed point is unique.

Proof of Theorem 13 can be done by following the lines of proofs of Theorems 8, 9, and 12. Hence, it is omitted.

Remark 14. Under the assumptions of Theorem 12, it can be proved that, for every $x \in X, \lim _{n \rightarrow \infty} T^{n} x=u$, where $u$ is the unique fixed point (i.e., the operator $T$ is Picard).

The contractions of classes (I) and (II) are quite general and many particular results can be concluded from Theorems $8-12$. Below we state some of these conclusions.

Corollary 15. Let $(X, d)$ be a complete metric space. Let $T$ : $X \rightarrow X$ be a continuous, weak triangular $\alpha$-admissible mapping such that

$$
\alpha(x, y) d(T x, T y) \leq k M(x, y), \quad \forall x, y \in X,
$$

where $0<k<1$ and $M(x, y)=\max \{d(x, y), d(x, T x)$, $d(y, T y),(1 / 2)[d(x, T y)+d(y, T x)]\}$. If there exists $x_{0} \in X$ such that $\alpha\left(x_{0}, T x_{0}\right) \geq 1$, then $T$ has a fixed point.

Proof. Proof is obvious by choosing $\psi(t)=t$ and $\phi(t)=k t$ in Theorem 8.

Corollary 16. Let $(X, d)$ be a complete metric space. Let $T$ : $X \rightarrow X$ be a continuous, weak triangular $\alpha$-admissible mapping such that

$$
\begin{aligned}
\alpha(x, y) d(T x, T y) \\
\leq \quad a d(x, y)+b d(x, T x)+c d(y, T y) \\
\quad+\frac{e}{2}[d(x, T y)+d(y, T x)],
\end{aligned}
$$

for all $x, y \in X$, where $0<a+b+c+e<1$. If there exists $x_{0} \in X$ such that $\alpha\left(x_{0}, T x_{0}\right) \geq 1$, then $T$ has a fixed point. 
Proof. Due to the fact that

$$
\begin{aligned}
& a d(x, y)+b d(x, T x)+c d(y, T y) \\
& \quad+\frac{e}{2}[d(x, T y)+d(y, T x)] \leq k M(x, y),
\end{aligned}
$$

the proof follows from Corollary 15.

\section{Fixed Points on Partially Ordered Metric Spaces}

It has been pointed out in some studies that some results in metric spaces endowed with a partial order can be concluded from the fixed point results related with $\alpha$-admissible maps on metric spaces (see $[9,10]$ ). In this section we give existence and uniqueness theorems on partially ordered metric spaces which can be regarded as consequences of the theorems presented in the previous section.

Recall that on a partially ordered set $(X, \preceq)$ a map $T$ : $X \rightarrow X$ is nondecreasing if it satisfies $T x \preceq T y$ for all $x, y \in X$ such that $x \preceq y$.

Definition 17. Let $(X, d, \preceq)$ be a metric space endowed with a partial order $\preceq$. If, for every nondecreasing sequence $\left\{x_{n}\right\} \subset X$ which converges to $x \in X$, there exists a subsequence $\left\{x_{n_{k}}\right\}$ of $\left\{x_{n}\right\}$ satisfying $x_{n_{k}} \preceq x$, then $(X, d, \preceq)$ is said to be regular.

Our first theorem contains the conditions for existence of a fixed point for a map of class (I) defined on a partially ordered metric space.

Theorem 18. Let $(X, d, \preceq)$ be a partially ordered complete metric space. Let $T: X \rightarrow X$ be a nondecreasing mapping such that

$$
\psi(d(T x, T y)) \leq \phi(M(x, y)), \quad \forall x, y \in X, \text { with } x \preceq y,
$$

where $\psi$ is an altering distance function, $\phi:[0,+\infty) \rightarrow$ $[0,+\infty)$ is a continuous function satisfying $\psi(t)>\phi(t)$ for all $t>0$, and $M(x, y)=\max \{d(x, y), d(x, T x)$, $d(y, T y),(1 / 2)[d(x, T y)+d(y, T x)]\}$. Assume that there exists $x_{0} \in X$ satisfying $x_{0} \preceq T x_{0}$ and that either $T$ is continuous or $(X, d, \preceq)$ is regular. Then $T$ has a fixed point.

Proof. Define the map $\alpha: X \times X \rightarrow[0, \infty)$ as

$$
\alpha(x, y)= \begin{cases}1 & \text { if } x \preceq y \text { or } y \leq x \\ 0 & \text { otherwise. }\end{cases}
$$

It is clear that $T$ satisfies

$$
\alpha(x, y) \psi(d(T x, T y)) \leq \phi(M(x, y)), \quad \forall x, y \in X,
$$

where $\alpha$ is defined in (56). Let $x_{0} \in X$ satisfy $x_{0} \preceq T x_{0}$. Then, $\alpha\left(x_{0}, T x_{0}\right) \geq 1$. On the other hand, since $T$ is nondecreasing, then $T$ is $\alpha$-admissible. Indeed,

$$
\begin{aligned}
& \alpha(x, y) \geq 1 \Longrightarrow x \preceq y \text { or } \\
& y \preceq x \Longrightarrow T x \preceq T y \text { or } \\
& T y \preceq T x \Longrightarrow \alpha(T x, T y) \geq 1 .
\end{aligned}
$$

Note also that if $x \preceq T x$ then $T x \preceq T^{2} x$, and hence $x \preceq T^{2} x$; that is, if $\alpha(x, T x) \geq 1$ then $\alpha\left(T x, T^{2} x\right) \geq 1$ and $\alpha\left(x, T^{2} x\right) \geq 1$. Similar conclusion can be done if $x \geq T x$. Therefore, $T$ is weak triangular $\alpha$-admissible. If $T$ is continuous, then $T$ satisfies the conditions of Theorem 8 and, hence, has a fixed point.

Suppose now that $X$ is regular. Then, every nondecreasing sequence $\left\{x_{n}\right\}$ which converges to $x$ has a subsequence $\left\{x_{n_{k}}\right\}$ for which $x_{n_{k}} \preceq x$ holds for all $k \in \mathbb{N}$. Hence, $\alpha\left(x_{n}, x_{n+1}\right) \geq 1$ implies $\alpha\left(x_{n_{k}}, x\right) \geq 1$ for all $k \in \mathbb{N}$. In other words, the set $X$ satisfies condition (A). By Theorem 9 , the mapping $T$ has a fixed point.

Analogously, we state conditions for existence of fixed points for maps from class (II) on partially ordered metric spaces.

Theorem 19. Let $(X, d, \preceq)$ be a partially ordered complete metric space. Let $T: X \rightarrow X$ be a nondecreasing mapping such that

$$
\psi(d(T x, T y)) \leq \phi(N(x, y)), \quad \forall x, y \in X, \text { with } x \preceq y,
$$

where $\psi$ is an altering distance function, $\phi:[0,+\infty) \rightarrow$ $[0,+\infty)$ is a continuous function satisfying $\psi(t)>\phi(t)$ for all $t>0$, and $N(x, y)=\max \{d(x, y),(1 / 2)[d(x, T x)+$ $d(y, T y)],(1 / 2)[d(x, T y)+d(y, T x)]\}$. Assume that there exists $x_{0} \in X$ satisfying $x_{0} \preceq T x_{0}$ and that either $T$ is continuous or $(X, d, \preceq)$ is regular. Then $T$ has a fixed point.

The uniqueness of a fixed point on partially ordered metric spaces requires an additional assumption on the set $X$. This assumption reads as follows.

(C) For all $x, y \in X$ there exists $z \in X$ which is comparable to both $x$ and $y$.

Theorem 20. Adding condition (C) to the hypothesis of Theorem 19 one obtains the uniqueness of the fixed point.

Proof. Note that if $\alpha$ is the map defined in (56) condition (C) is equivalent to condition (B). Indeed, assume that condition (B) holds. Then for all $x, y \in X$ there exists $z \in X$ such that $\alpha(x, z) \geq 1$ and $\alpha(y, z) \geq 1$, where $\alpha$ is defined in (56). This means that both $x$ and $y$ are comparable to $z$; that is, condition (C) also holds. If, on the other hand, condition (C) is satisfied then for all $x, y \in X$ there exists $z \in X$ which is comparable to both $x$ and $y$. Then $\alpha(x, z)=1 \geq 1$ and $\alpha(y, z)=1 \geq 1$; that is, condition (B) is also satisfied. Hence, by Theorem 12, the fixed point of the map $T$ is unique.

Some consequences of the results stated above can be easily concluded. We next present two of them.

Corollary 21. Let $(X, d, \preceq)$ be a partially ordered and complete metric space. Let $T: X \rightarrow X$ be nondecreasing mapping such that

$$
d(T x, T y) \leq k M(x, y), \quad \forall x, y \in X \text { with } x \preceq y,
$$

where $0<k<1$ and $M(x, y)=\max \{d(x, y), d(x, T x)$, $d(y, T y),(1 / 2)[d(x, T y)+d(y, T x)]\}$. Assume that there exists 
$x_{0} \in X$ such that $x_{0} \preceq T x_{0}$. If $T$ is continuous or $X$ is regular, then $T$ has a fixed point.

Proof. Proof is obvious by choosing $\psi(t)=t$ and $\phi(t)=k t$ in Theorem 18.

Corollary 22. Let $(X, d, \preceq)$ be a partially ordered and complete metric space. Let $T: X \rightarrow X$ be nondecreasing mapping such that

$$
\begin{gathered}
d(T x, T y) \\
\leq a d(x, y)+b d(x, T x)+c d(y, T y) \\
+\frac{e}{2}[d(x, T y)+d(y, T x)], \\
\forall x, y \in X \text { with } x \preceq y,
\end{gathered}
$$

where $0<a+b+c+e<1$. Assume that there exists $x_{0} \in X$ such that $x_{0} \preceq T x_{0}$. If $T$ is continuous or $X$ is regular, then $T$ has a fixed point.

Proof. Due to the fact that

$$
\begin{aligned}
& a d(x, y)+b d(x, T x)+c d(y, T y) \\
& \quad+\frac{e}{2}[d(x, T y)+d(y, T x)] \leq k M(x, y),
\end{aligned}
$$

the proof follows from Corollary 21.

Our last result is a consequence of Theorem 13 on partially ordered metric spaces and follows easily by using the function $\alpha$ defined in (56). It is actually the main result of Yan et al. [7].

Corollary 23. Let $(X, d, \preceq)$ be a partially ordered and complete metric space. Let $T: X \rightarrow X$ be a nondecreasing mapping such that

$$
\begin{gathered}
\psi(d(T x, T y)) \leq \phi(d(x, y)), \\
\forall x, y \in X \text { for which } x \preceq y,
\end{gathered}
$$

where $\psi$ is an altering distance function and $\phi:[0,+\infty) \rightarrow$ $[0,+\infty)$ is a continuous function satisfying $\psi(t)>\phi(t)$ for all $t>0$. Assume either that $T$ is continuous or that $X$ is regular. If there exists $x_{0} \in X$ such that $x_{0} \preceq T x_{0}$, then $T$ has a fixed point. If, in addition, $X$ satisfies condition (C), then the fixed point is unique.

\section{Application to Ordinary Differential Equations}

In this section, we discuss application of our results to existence and uniqueness of solutions of boundary value problems. We present two examples, first of which has been inspired by [1]. Specifically, we study the existence and uniqueness of a solution for the following first-order periodic boundary value problem:

$$
\begin{aligned}
& u^{\prime}(t)=f(t, u(t)), \quad \text { if } t \in I=[0, T], \\
& u(0)=u(T),
\end{aligned}
$$

where $T>0$ and $f: I \times \mathbb{R} \rightarrow \mathbb{R}$ is a continuous function. Let $C(I)$ denote the space of continuous functions defined on I. Clearly, the function

$$
d(x, y)=\sup _{t \in I}|x(t)-y(t)|, \quad x, y \in C(I)
$$

defines a metric on $C(I)$. Moreover, $(C(I), d)$ is a complete metric space. Define also a partial order on $C(I)$ by

$$
x, y \in C(I), \quad x \leq y \Longleftrightarrow x(t) \leq y(t) \quad \forall t \in I .
$$

It is easy to see that $C(I)$ satisfies condition (C). Indeed, for $x, y \in C(I)$, we have $x \leq z$ and $y \leq z$, where

$$
z=\max _{t \in I}\{x(t), y(t)\}= \begin{cases}x(t) & \text { if } x(t) \geq y(t) \\ y(t) & \text { if } y(t) \geq x(t)\end{cases}
$$

is also in $C(I)$ whenever $x, y \in C(I)$. Nieto and RodríguezLópez [1] proved that $(C(I), d, \leq)$, where the metric $d$ and the partial order $\leq$ are defined above, satisfies regularity condition given in Definition 17. We now define a lower solution for the problem (64).

Definition 24. A lower solution for (64) is a function $\beta \in$ $C^{1}(I)$ satisfying

$$
\begin{aligned}
& \beta^{\prime}(t) \leq f(t, \beta(t)), \quad \text { for } t \in I, \\
& \beta(0) \leq \beta(T) .
\end{aligned}
$$

Our next theorem gives conditions for existence and uniqueness of solution to the problem (64).

Theorem 25. Let the function $f$ in the problem (64) be continuous and let

$$
0 \leq f(t, y)+\lambda y-[f(t, x)+\lambda x] \leq \gamma\left(\frac{(x-y)^{2}}{(x-y)^{2}+1}\right)^{1 / 2}
$$

hold for $x, y \in \mathbb{R}$ with $y \geq x$ and for some $\lambda, \gamma>0$ satisfying

$$
\gamma \leq\left(\frac{2 \lambda\left(e^{\lambda T}-1\right)}{T\left(e^{\lambda T}+1\right)}\right)^{1 / 2} .
$$

If the problem (64) has a lower solution, then it has a unique solution.

Proof. Rewrite the problem (64) as

$$
\begin{gathered}
u^{\prime}(t)+\lambda u(t)=f(t, u(t))+\lambda u(t), \quad \text { for } t \in I=[0, T], \\
u(0)=u(T) .
\end{gathered}
$$

Clearly, the problem (64) is equivalent to the integral equation

$$
u(t)=\int_{0}^{T} G(t, s)[f(s, u(s))+\lambda u(s)] d s,
$$


where $G(t, s)$ is the Green function given by

$$
G(t, s)= \begin{cases}\frac{e^{\lambda(T+s-t)}}{e^{\lambda T}-1}, & 0 \leq s<t \leq T, \\ \frac{e^{\lambda(s-t)}}{e^{\lambda T}-1}, & 0 \leq t<s \leq T\end{cases}
$$

Define the map $\mathscr{A}: C(I) \rightarrow C(I)$ by

$$
(\mathscr{A} u)(t)=\int_{0}^{T} G(t, s)[f(s, u(s))+\lambda u(s)] d s, \quad t \in I .
$$

It is obvious that a fixed point $u \in C(I)$ of $\mathscr{A}$ is a solution of (64). We will show that the mapping $A$ satisfies the conditions of Corollary 23.

First, note that $\mathscr{A}$ is nondecreasing by the hypothesis; that is, for $u \geq v$,

$$
f(t, u)+\lambda u \geq f(t, v)+\lambda v
$$

And, hence,

$$
\begin{aligned}
(\mathscr{A} u)(t) & =\int_{0}^{T} G(t, s)[f(s, u(s))+\lambda u(s)] d s \\
& \geq \int_{0}^{T} G(t, s)[f(s, v(s))+\lambda v(s)] d s=(\mathscr{A} v)(t)
\end{aligned}
$$

since $G(t, s)>0$ for $(t, s) \in I \times I$.

Note also that, for $u \geq v$, we have

$$
\begin{aligned}
d(\mathscr{A} u, \mathscr{A} v) & =\sup _{t \in I}|(\mathscr{A} u)(t)-(\mathscr{A} v)(t)| \\
& =\sup _{t \in I}((\mathscr{A} u)(t)-(\mathscr{A} v)(t)) \\
& =\sup _{t \in I} \int_{0}^{T} G(t, s)[f(s, u(s))+\lambda u(s) \\
& -f(s, v(s))+\lambda v(s)] d s \\
\leq & \sup _{t \in I} \int_{0}^{T} G(t, s) \gamma \sqrt{\frac{[u(s)-v(s)]^{2}}{[u(s)-v(s)]^{2}+1}} d s .
\end{aligned}
$$

Employing the Cauchy-Schwarz inequality for the last integral, we obtain

$$
\begin{aligned}
& \int_{0}^{T} G(t, s) \gamma \sqrt{\frac{(u(s)-v(s))^{2}}{(u(s)-v(s))^{2}+1}} d s \\
& \quad \leq\left(\int_{0}^{T} G(t, s)^{2} d s\right)^{1 / 2}\left(\int_{0}^{T} \gamma^{2} \frac{[u(s)-v(s)]^{2}}{[u(s)-v(s)]^{2}+1} d s\right)^{1 / 2} .
\end{aligned}
$$

The first integral on the right-hand side can be calculated easily and gives

$$
\begin{aligned}
\int_{0}^{T} G(t, s)^{2} d s & =\int_{0}^{t} G(t, s)^{2} d s+\int_{t}^{T} G(t, s)^{2} d s \\
& =\int_{0}^{t} \frac{e^{2 \lambda(T+s-t)}}{\left(e^{\lambda T}-1\right)^{2}} d s+\int_{t}^{T} \frac{e^{2 \lambda(s-t)}}{\left(e^{\lambda T}-1\right)^{2}} d s \\
& =\frac{e^{2 \lambda T}-1}{2 \lambda\left(e^{\lambda T}-1\right)^{2}} \\
& =\frac{e^{\lambda T}+1}{2 \lambda\left(e^{\lambda T}-1\right)} .
\end{aligned}
$$

For the second integral in (78) we obtain the following estimate:

$$
\begin{aligned}
\int_{0}^{T} \gamma^{2} \frac{[u(s)-v(s)]^{2}}{[u(s)-v(s)]^{2}+1} d s & \leq \gamma^{2} \sup _{t \in I} \frac{|u(t)-v(t)|^{2}}{|u(t)-v(t)|^{2}+1} \cdot T \\
& =\gamma^{2} \frac{d(u, v)^{2}}{d(u, v)^{2}+1} T
\end{aligned}
$$

Making use of the inequalities in (77), (78), and (80) and the integral in (79) we deduce

$$
\begin{aligned}
& d(\mathscr{A} u, \mathscr{A} v) \\
& \quad \leq\left(\frac{e^{\lambda T}+1}{2 \lambda\left(e^{\lambda T}-1\right)}\right)^{1 / 2} \cdot\left(\gamma^{2} \frac{d(u, v)^{2}}{d(u, v)^{2}+1} T\right)^{1 / 2} \\
& \quad=\left(\frac{e^{\lambda T}+1}{2 \lambda\left(e^{\lambda T}-1\right)}\right)^{1 / 2} \gamma\left(\frac{d(u, v)^{2}}{d(u, v)^{2}+1}\right)^{1 / 2} \sqrt{T} .
\end{aligned}
$$

And, consequently,

$$
d(\mathscr{A} u, \mathscr{A} v)^{2} \leq \gamma^{2} \frac{e^{\lambda T}+1}{2 \lambda\left(e^{\lambda T}-1\right)} \frac{d(u, v)^{2}}{d(u, v)^{2}+1} T
$$

This last inequality can be also written as

$$
\begin{gathered}
\left(2 \lambda\left(e^{\lambda T}-1\right)\right)\left(d(u, v)^{2}+1\right) d(\mathscr{A} u, \mathscr{A} v)^{2} \\
\leq \gamma^{2}\left(e^{\lambda T}+1\right) d(u, v)^{2} T
\end{gathered}
$$

since $\lambda$ is positive. The constant $\gamma$, on the other hand, satisfies

$$
\gamma \leq\left(\frac{2 \lambda\left(e^{\lambda T}-1\right)}{T\left(e^{\lambda T}+1\right)}\right)^{1 / 2}
$$

and, therefore, inequality (83) implies

$$
\begin{gathered}
\left(2 \lambda\left(e^{\lambda T}-1\right)\right)\left(d(u, v)^{2}+1\right) d(\mathscr{A} u, \mathscr{A} v)^{2} \\
\leq \frac{2 \lambda\left(e^{\lambda T}-1\right)}{T\left(e^{\lambda T}+1\right)}\left(e^{\lambda T}+1\right) d(u, v)^{2} T
\end{gathered}
$$


and, thus,

$$
d(\mathscr{A} u, \mathscr{A} v)^{2} \leq \frac{d(u, v)^{2}}{d(u, v)^{2}+1}
$$

Define the functions $\psi$ and $\phi$ as $\psi(t)=t^{2}$ and $\phi(t)=t^{2} /\left(t^{2}+\right.$ $1)$. It is clear that $\psi$ is an altering distance function and also that $\psi(t)>\phi(t)$ for all $t>0$. Then (86) becomes

$$
\psi(d(\mathscr{A} u, \mathscr{A} v)) \leq \phi(d(u, v))
$$

for all $u \geq v$; that is, $\mathscr{A}$ satisfies the contractive condition of Corollary 23. Finally, let $\beta(t)$ be a lower solution for (86). We will show that $\beta \leq \mathscr{A} \beta$. Multiplying the inequality

$$
\beta^{\prime}(t)+\lambda \beta(t) \leq f(t, \beta(t))+\lambda \beta(t), \quad \text { for } t \in I,
$$

by $e^{\lambda t}$, we obtain

$$
\left(\beta(t) e^{\lambda t}\right)^{\prime} \leq[f(t, \beta(t))+\lambda \beta(t)] e^{\lambda t}, \quad \text { for } t \in I,
$$

which upon integration gives

$$
\beta(t) e^{\lambda t} \leq \beta(0)+\int_{0}^{t}[f(s, \beta(s))+\lambda \beta(s)] e^{\lambda s} d s, \quad \text { for } t \in I .
$$

Since $\beta(0) \leq \beta(T)$, the last inequality implies

$$
\begin{aligned}
\beta(0) e^{\lambda t} & \leq \beta(T) e^{\lambda T} \\
& \leq \beta(0)+\int_{0}^{T}[f(s, \beta(s))+\lambda \beta(s)] e^{\lambda s} d s,
\end{aligned}
$$

and, hence,

$$
\beta(0) \leq \int_{0}^{T} \frac{e^{\lambda s}}{e^{\lambda T}-1}[f(s, \beta(s))+\lambda \beta(s)] d s .
$$

From this inequality and inequality (90) we obtain

$$
\begin{aligned}
\beta(t) e^{\lambda t} \leq & \int_{0}^{t} \frac{e^{\lambda(T+s)}}{e^{\lambda T}-1}[f(s, \beta(s))+\lambda \beta(s)] d s \\
& +\int_{t}^{T} \frac{e^{\lambda s}}{e^{\lambda T}-1}[f(s, \beta(s))+\lambda \beta(s)] d s,
\end{aligned}
$$

and, consequently,

$$
\begin{aligned}
\beta(t) \leq & \int_{0}^{t} \frac{e^{\lambda(T+s-t)}}{e^{\lambda T}-1}[f(s, \beta(s))+\lambda \beta(s)] d s \\
& +\int_{t}^{T} \frac{e^{\lambda(s-t)}}{e^{\lambda T}-1}[f(s, \beta(s))+\lambda \beta(s)] d s \\
= & \int_{0}^{T} G(t, s)[f(s, \beta(s))+\lambda \beta(s)] d s \\
= & (\mathscr{A} \beta)(t), \quad \text { for } t \in I .
\end{aligned}
$$

We have shown that all conditions of Corollary 23 hold. Therefore $\mathscr{A}$ has a unique fixed point, or, equivalently, the problem (64) has a unique solution.
As a second example we discuss the existence and uniqueness of solution for a second-order boundary value problem. More precisely, we consider

$$
\begin{gathered}
-\frac{d^{2} u}{d t^{2}}=f(t, u), \quad u \in I=[0, \infty), t \in[0,1], \\
u(0)=u(1)=0 .
\end{gathered}
$$

The problem (95) is equivalent to the integral equation

$$
u(t)=\int_{0}^{1} G(t, s) f(s, u(s)) d s, \quad t \in[0,1],
$$

where $G(t, s)$ is the Green function given by

$$
G(t, s)= \begin{cases}t(1-s), & 0 \leq t<s \leq 1 \\ s(1-t), & 0 \leq s<t \leq 1\end{cases}
$$

In what follows, our last theorem gives conditions for the existence and uniqueness of solution to the problem (95) or, equivalently, the problem (96).

Theorem 26. Let $f:[0,1] \times \mathbb{R} \rightarrow[0, \infty)$ be a continuous function which is nondecreasing in its second variable. Suppose that for all $u, v \in \mathbb{R}$ satisfying $u \leq v$, there exists a constant $0<\gamma<8$ such that

$$
f(t, v)-f(t, u) \leq \gamma\left(\frac{(u-v)^{2}}{(u-v)^{2}+1}\right)^{1 / 2}
$$

Then the problem (95) has a unique nonnegative solution.

Proof. Consider the space $Y=\{u \in C[0,1]: u(t) \geq 0\}$ and define a metric $d(u, v)=\sup \{|u(t)-v(t)|: t \in[0,1]\}$ as usual. Obviously, $(Y, d)$ is a complete metric space. Define also the usual partial order on $Y$; that is,

$$
u \leq v \Longrightarrow u(t) \leq v(t) \quad \forall t \in[0,1]
$$

As in the previous example, a solution of the problem (95) is a fixed point of the operator $\mathscr{A}: Y \rightarrow Y$ defined by

$$
(\mathscr{A} u)(t)=\int_{0}^{1} G(t, s) f(s, u(s)) d s, \quad t \in[0,1]
$$

where $G(t, s)$ is the Green function in (97). Since $f$ is nondecreasing with respect to its second variable, then for $u, v \in Y$ with $v \geq u$ we have

$$
\begin{aligned}
(\mathscr{A} v)(t) & =\int_{0}^{1} G(t, s) f(s, v(s)) d s \\
& \geq \int_{0}^{1} G(t, s) f(s, u(s)) d s \\
& =(\mathscr{A} u)(t),
\end{aligned}
$$


for all $t \in[0,1]$; that is, $\mathscr{A}$ is nondecreasing. On the other hand, using the condition in (98), we estimate

$$
\begin{aligned}
d & (\mathscr{A} v, \mathscr{A} u) \\
& =\sup _{t \in[0,1]}|(\mathscr{A} v)(t)-(\mathscr{A} u)(t)| \\
& =\sup _{t \in[0,1]}((\mathscr{A} v)(t)-(\mathscr{A} u)(t)) \\
& =\sup _{t \in[0,1]} \int_{0}^{1} G(t, s)[f(s, v(s))-f(s, u(s))] d s \\
& \leq \sup _{t \in[0,1]} \int_{0}^{1} G(t, s) \gamma \sqrt{\frac{(v(s)-u(s))^{2}}{(v(s)-u(s))^{2}+1}} d s \\
& \leq \gamma \sup _{t \in[0,1]} \sqrt{\frac{|v(t)-u(t)|^{2}}{|v(t)-u(t)|^{2}+1}} \sup _{t \in[0,1]} \int_{0}^{1} G(t, s) d s .
\end{aligned}
$$

Moreover, calculating the integral of Green's function, which is

$$
\int_{0}^{1} G(t, s) d s=-\frac{t^{2}}{2}+\frac{t}{2}
$$

we obtain

$$
\sup _{t \in[0,1]} \int_{0}^{1} G(t, s) d s=\frac{1}{8} .
$$

Therefore, inequality (102) and the assumption $0<\gamma<8$ result in

$$
\begin{aligned}
d(\mathscr{A} v, \mathscr{A} u) & \leq \frac{\gamma}{8} \sup _{t \in[0,1]} \sqrt{\frac{|v(t)-u(t)|^{2}}{|v(t)-u(t)|^{2}+1}} \\
& \leq \sup _{t \in[0,1]} \sqrt{\frac{|v(t)-u(t)|^{2}}{|v(t)-u(t)|^{2}+1}} \\
& =\sqrt{\frac{d(v, u)^{2}}{d(v, u)^{2}+1}},
\end{aligned}
$$

or, shortly, in

$$
d(\mathscr{A} v, \mathscr{A} u)^{2} \leq \frac{d(v, u)^{2}}{d(v, u)^{2}+1} .
$$

Define the functions $\psi(t)=t^{2}, \phi(t)=t^{2} /\left(t^{2}+1\right)$ as in the previous example, and note that these functions satisfy the conditions of Corollary 23. Inequality (106) becomes

$$
\psi(d(\mathscr{A} v, \mathscr{A} u)) \leq \phi(d(v, u)) .
$$

Finally, since both functions $f$ and $G$ are nonnegative, we get

$$
(\mathscr{A} 0)(t)=\int_{0}^{1} G(t, s) f(s, 0) d s \geq 0 .
$$

Therefore, all the conditions of Corollary 23 hold and, hence, the operator $\mathscr{A}$ has a unique fixed point; that is, the problem (95) has a unique nonnegative solution.

\section{Conflict of Interests}

The authors declare that there is no conflict of interests regarding the publication of this paper.

\section{Authors' Contribution}

All authors contributed equally and significantly in writing this paper. All authors read and approved the final paper.

\section{References}

[1] J. J. Nieto and R. Rodríguez-López, "Contractive mapping theorems in partially ordered sets and applications to ordinary differential equations," Order, vol. 22, no. 3, pp. 223-239, 2005.

[2] J. Harjani and K. Sadarangani, "Fixed point theorems for weakly contractive mappings in partially ordered sets," Nonlinear Analysis: Theory, Methods \& Applications, vol. 71, no. 7-8, pp. 34033410, 2009.

[3] J. Harjani and K. Sadarangani, "Generalized contractions in partially ordered metric spaces and applications to ordinary differential equations," Nonlinear Analysis: Theory, Methods \& Applications, vol. 72, no. 3-4, pp. 1188-1197, 2010.

[4] J. Caballero, J. Harjani, and K. Sadarangani, "Contractive-like mapping principles in ordered metric spaces and application to ordinary differential equations," Fixed Point Theory and Applications, vol. 2010, Article ID 916064, 14 pages, 2010.

[5] A. Amini-Harandi and H. Emami, "A fixed point theorem for contraction type maps in partially ordered metric spaces and application to ordinary differential equations," Nonlinear Analysis: Theory, Methods \& Applications, vol. 72, no. 5, pp. 2238-2242, 2010.

[6] B. Samet, C. Vetro, and P. Vetro, "Fixed point theorems for $\alpha$ - $\psi$-contractive type mappings," Nonlinear Analysis: Theory, Methods and Applications, vol. 75, no. 4, pp. 2154-2165, 2012.

[7] F. Yan, Y. Su, and Q. Feng, "A new contraction mapping principle in partially ordered metric spaces and applications to ordinary differential equations," Fixed Point Theory and Applications, vol. 2012, article 152, 2012.

[8] M. S. Khan, M. Swaleh, and S. Sessa, "Fixed point theorems by altering distances between the points," Bulletin of the Australian Mathematical Society, vol. 30, no. 1, pp. 1-9, 1984.

[9] E. Karapınar, P. Kumam, and P. Salimi, "On $\alpha \psi$-Meir-Keeler contractive mappings," Fixed Point Theory and Applications, vol. 2013, article 94, 2013.

[10] E. Karapınar and B. Samet, "Generalized $\alpha-\psi$ contractive type mappings and related fixed point theorems with applications," Abstract and Applied Analysis, vol. 2012, Article ID 793486, 17 pages, 2012. 


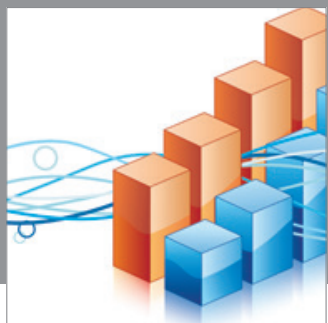

Advances in

Operations Research

mansans

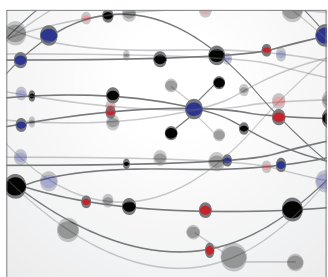

The Scientific World Journal
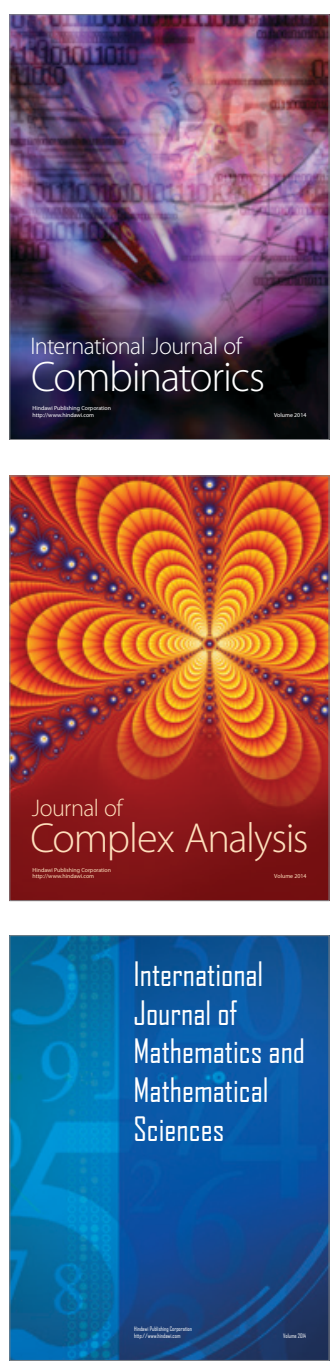
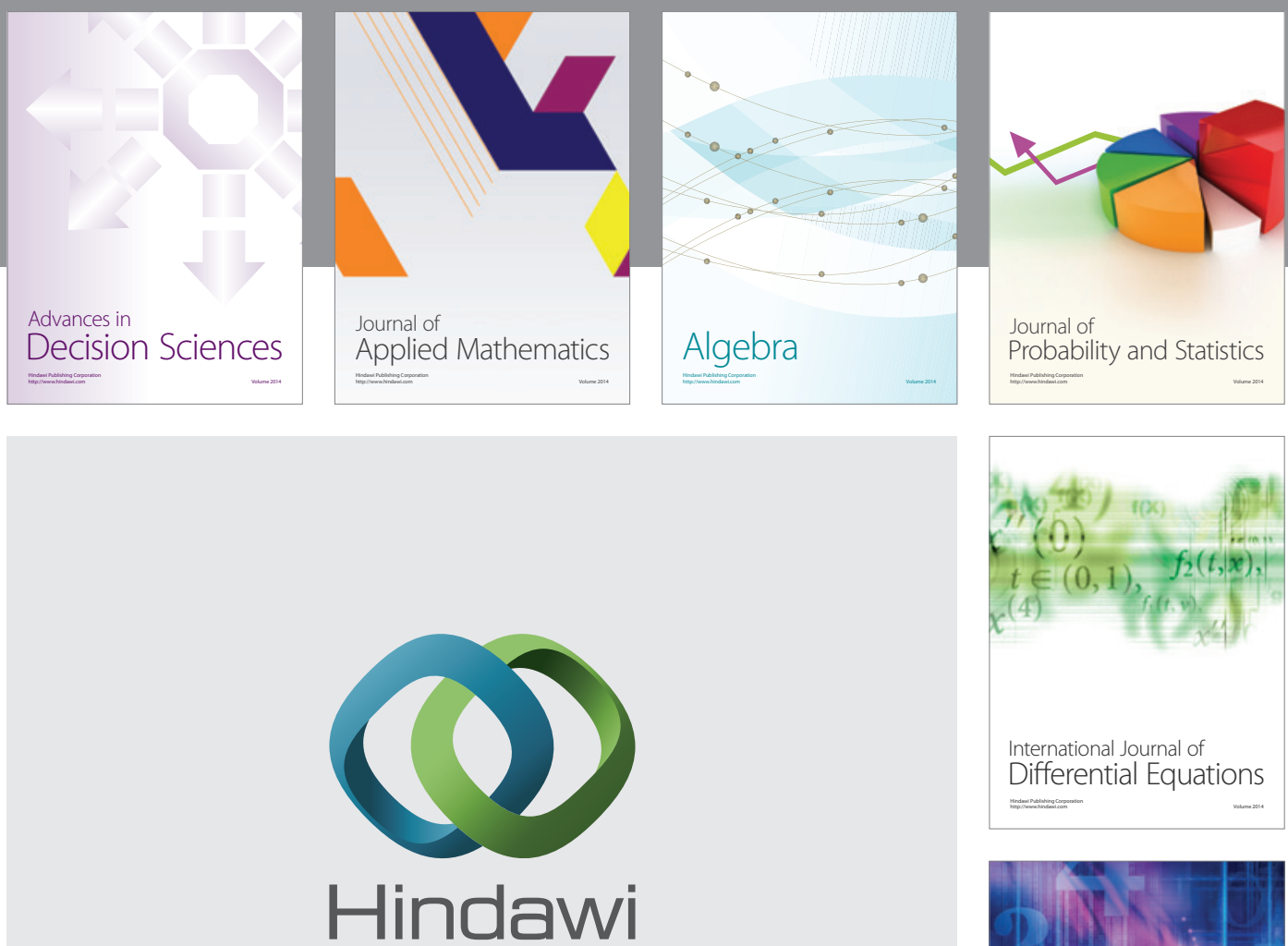

Submit your manuscripts at http://www.hindawi.com
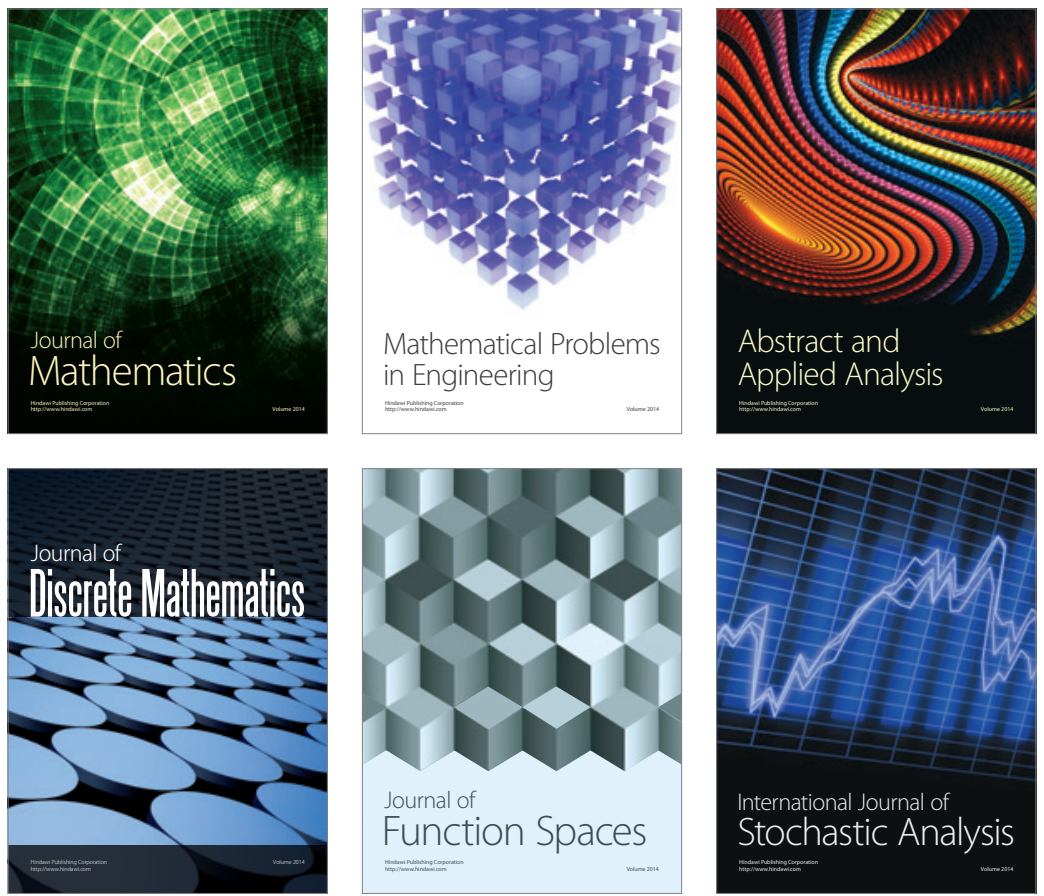

Journal of

Function Spaces

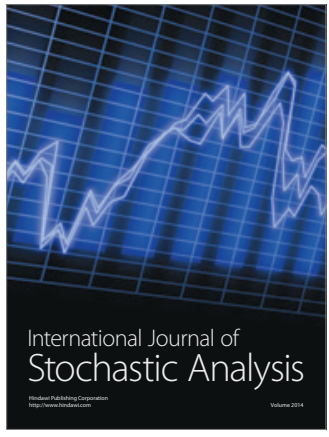

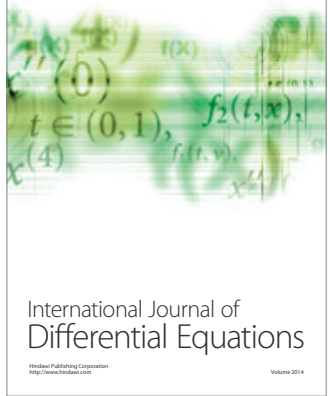
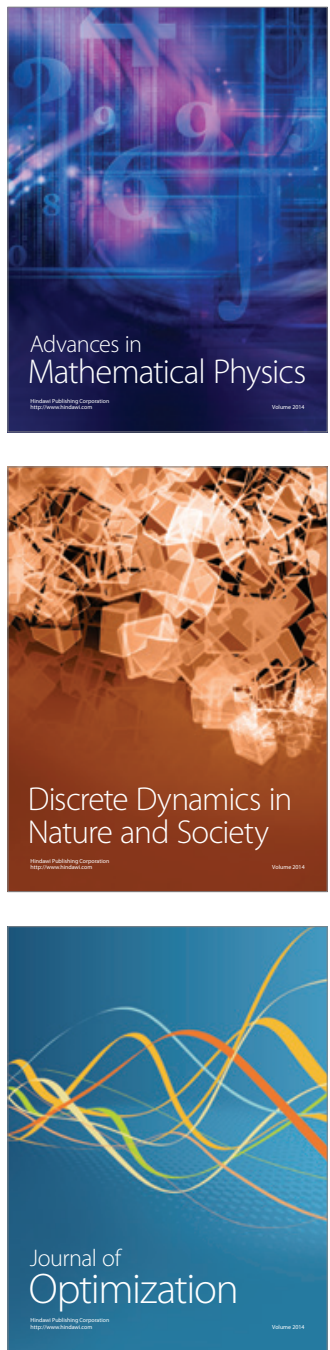